\title{
Anomalous Temperature Dependence of the Supercurrent Through a Chaotic Josephson Junction
}

\author{
P. W. BROUWER and C. W. J. BEENAKKER \\ Instituut-Lorentz, University of Leiden, P.O. Box 9506, 2300 RA Leiden, The Netherlands
}

\begin{abstract}
We calculate the supercurrent through a Josephson junction consisting of a phase-coherent metal particle (quantum dot), weakly coupled to two superconductors. The classical motion in the quantum dot is assumed to be chaotic on time scales greater than the ergodic time $\tau_{\text {ela }}$, which itself is much smaller than the mean dwell time $\tau_{\mathrm{dwell}}$. The excitation spectrum of the Josephson junction has a gap $E_{\text {gap }}$, which can be less than the gap $\Delta$ in the bulk superconductors. The average supercurrent is computed in the ergodic regime $\tau_{\mathrm{el} g} \ll \hbar / \Delta$, using random-matrix theory, and in the non-ergodic regime $\tau_{\mathrm{etg}} \gg \hbar / \Delta$, using a semiclassical relation between the supercurrent and dwell-time distribution. In contrast to conventional Josephson junctions, raising the temperature above the excitation gap does not necessarily lead to an exponential suppression of the supercurrent. Instead, we find a temperature regime between $E_{\mathrm{gap}}$ and $\Delta$ where the supercurrent decreases logarithmically with temperature. This anomalously weak temperature dependence is caused by long-range correlations in the excitation spectrum, which extend over an energy range $\hbar / \tau_{\mathrm{clg}}$ greater than $E_{\mathrm{gap}} \simeq \hbar / \tau_{\mathrm{dwcll}}$. A similar logarithmic temperature dependence of the supercurrent was discovered by Aslamazov, Larkın and Ovchinnikov in a Josephson junction consisting of a disordered metal between two tunnel barriers. (C) 1997 Elsevier Scrence Ltd
\end{abstract}

\section{INTRODUCTION}

The dissipationless flow of a current through a superconductor-normal-metal-superconductor (SNS) junction is a fundamental demonstration of the 'proximity effect': a normal metal borrows characteristic properties from a nearby superconductor. The energy gap $\Delta$ in the bulk induces a suppression of the density of states inside the normal metal near the Fermi level, depending on the phase difference $\phi$ between the superconductors. The resulting $\phi$-dependence of the free energy $F$ implies the flow of a current $I=(2 e / \hbar) \mathrm{d} F / \mathrm{d} \phi$ in equilibrium. In contrast to the original Josephson effect in tunnel junctions, the separation of the superconductors in an SNS junction can be much greater than the superconducting coherence length. Recent experiments on mesoscopic Josephson junctions [1-6] have revived theoretical interest in this subject [7-9], which goes back to work by Kulik [10] and Aslamasov, Larkin and Ovchinnikov [11]. (For more references, see the review [12].)

In this paper, we consider the case when the normal region consists of a chaotic quantum dot. A quantum dot is a small metal particle within which the motion is phase coherent, weakly coupled to the superconductors by means of point contacts. We assume that the classical dynamics in the quantum dot are chaotic on time scales longer than the time $\tau_{\mathrm{erg}}$ needed for ergodic exploration of the phase space of the quantum dot. (In order of magnitude, $\tau_{\mathrm{erg}} \simeq L / v_{\Gamma}$ for a quantum dot of size $L$ without impurities, where $v_{\mathrm{F}}$ is the Fermi velocity.) On energy scales smaller than $\hbar / \tau_{\text {erg }}$, the spectral statistics of a chaotic quantum dot is described by random-matrix theory $[13,14]$. On larger energy scales, the non-ergodic dynamics on time scales below $\tau_{\text {erg }}$ become dominant [15]. The condition of weak coupling 
We now perform a partial integration and close the integration contour in the upper half of the complex plane. The integrand has poles at the Matsubara frequencies $i \omega_{n}=(2 n+$ 1)i $\pi k T$. Summing over the residues, one finds that

$$
I=-\frac{e}{\hbar} 2 k T \frac{\mathrm{d}}{\mathrm{d} \phi} \sum_{n=0}^{\infty} \ln \operatorname{det}\left[1-\mathscr{S}_{\mathrm{A}}\left(\mathrm{i} \omega_{n}\right) \mathscr{S}_{\mathrm{N}}\left(\mathrm{i} \omega_{n}\right)\right]
$$

This equation is the starting point for our evaluation of the average supercurrent through a chaotic Josephson junction.

\section{SUPERCURRENT THROUGH A CHAOTIC JOSEPHSON JUNCTION}

We consider the case when the normal region has a chaotic classical dynamics on time

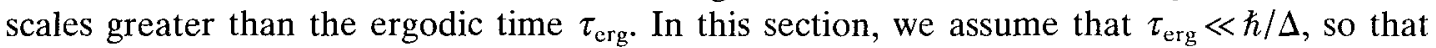
we may use random-matrix theory to evaluate the ensemble average of the supercurrent. We postpone to Section 5, a discussion of the regime $\tau_{\text {erg }} \geq \hbar / \Delta$, in which the non-ergodic dynamics on time scales shorter than $\tau_{\text {erg }}$ starts to play a role. We assume that the normal metal is weakly coupled to the superconductors, so that the mean dwell time $\tau_{\text {dwell }} \gg \tau_{\text {erg. No }}$. No assumption is made regarding the relative magnitudes of $\tau_{\text {dwell }}$ and $\hbar / \Delta$.

We use a relationship [24,25] between the scattering matrix $S$ of the normal metal and its Hamiltonian $H$

$$
S(\varepsilon)=1-2 \pi \mathrm{i} W^{\dagger}\left(\varepsilon-H+\mathrm{i} \pi W W^{\dagger}\right)^{-1} W .
$$

The Hamiltonian $H$ (representing the isolated normal metal region) is taken from the Gaussian ensemble of random-matrix theory [26],

$$
P(H) \propto \exp \left(-\frac{1}{4} M \lambda^{-2} \operatorname{tr} H^{2}\right)
$$

where $M$ is the dimension of $H$ (taken to infinity at the end) and $\lambda$ is a parameter that determines the average level spacing $\delta=\lambda \pi / 2 M$ of the excitation spectrum in the normal region. (This spacing $\delta$ is half the level spacing of $H$, because it combines electron and hole levels together.) The matrix $H$ is real and symmetric. The coupling matrix $W$ is an $M \times N$ matrix $[27,28]$ with elements

$$
W_{m n}=\frac{1}{\pi} \delta_{m n}(2 M \delta)^{1 / 2}\left(2 \Gamma_{n}^{-1}-1-2 \Gamma_{n}^{-1} \sqrt{1-\Gamma_{n}}\right)^{1 / 2}
$$

Here $\Gamma_{n}$ is the transmission probability of mode $n$ in the contacts to the superconductor. For ballistic contacts $\Gamma_{n}=1$, while $\Gamma_{n} \ll 1$ for tunneling contacts.

We now substitute from equation (9) into equation (1) and then substitute $\mathscr{S}_{\mathrm{N}}$ into equation (8) for the supercurrent. Using also equations (2a) and (2b), we find after some straightforward matrix algebra that

$$
I=-\frac{2 e}{\hbar} 2 k T \frac{\mathrm{d}}{\mathrm{d} \phi} \sum_{n=0}^{\infty} \ln \operatorname{det}\left[\mathrm{i} \omega_{n}-\mathscr{H}+\mathscr{W}\left(\mathrm{i} \omega_{n}\right)\right],
$$


where we have introduced the $2 M \times 2 M$ matrices

$$
\begin{gathered}
\mathscr{H}=\left(\begin{array}{cc}
H & 0 \\
0 & -H
\end{array}\right), \\
\mathscr{W}(\varepsilon)=\frac{\pi \Delta}{\sqrt{\Delta^{2}-\varepsilon^{2}}}\left(\begin{array}{cc}
(\varepsilon / \Delta) W W^{\mathrm{T}} & W \mathrm{e}^{\mathrm{II}} W^{\mathrm{T}} \\
W \mathrm{e}^{-1 \Phi} W^{\mathrm{T}} & (\varepsilon / \Delta) W W^{\mathrm{T}}
\end{array}\right) .
\end{gathered}
$$

The matrix $\mathscr{H}-\mathscr{W}(\varepsilon)$ is the effective Hamiltonian of Refs $[18,29]$ (where the regime $\varepsilon \ll \Delta$ was considered, in which the $\varepsilon$-dependence of $\mathscr{W}(\varepsilon)$ can be neglected).

We define the $2 M \times 2 M$ Green function

$$
\mathscr{G}(\varepsilon)=[\varepsilon-\mathscr{H}+\mathscr{W}(\varepsilon)]^{-1},
$$

which determines the density of states according to

$$
\rho(\varepsilon)=-\pi^{-1} \operatorname{Im} \operatorname{tr} \mathscr{G}(\varepsilon+\mathrm{i} 0) .
$$

Equation (15) is equivalent to equation (4). The expression for the supercurrent in terms of $G(\varepsilon)$ is

$$
\begin{aligned}
I & =\frac{2 e}{\hbar} 2 k T \frac{\mathrm{d}}{\mathrm{d} \phi} \sum_{n=0}^{\infty} \ln \operatorname{det} \mathscr{G}\left(\mathrm{i} \omega_{n}\right) \\
& =-\frac{2 e}{\hbar} 2 k T \sum_{n=0}^{\infty} \operatorname{tr} \mathscr{G}\left(\mathrm{i} \omega_{n}\right) \frac{\mathrm{d}}{\mathrm{d} \phi} \mathscr{W}\left(\mathrm{i} \omega_{n}\right) .
\end{aligned}
$$

The average supercurrent follows from the average Green function $\langle\mathscr{G}(\varepsilon)\rangle$, since $\mathscr{W}$ is a fixed matrix. The average over the random Hamiltonian $H$ (determining $\mathscr{G}$ ) is done with the help of the diagrammatic technique of Refs $[30,31]$. We consider the regime $M, N,|\varepsilon| / \delta \gg 1$, in which only planar diagrams need to be considered. Resummation of these diagrams leads to a self-consistency equation which is similar to Pastur's equation [32]

$$
\langle\mathscr{G}(\varepsilon)\rangle=\left[\varepsilon+\mathscr{W}(\varepsilon)-\left(\lambda^{2} / M\right) \mathscr{P}(\varepsilon) \otimes \mathbb{1}_{M}\right]^{-1} .
$$

The symbol $\otimes$ indicates the direct product between the $M \times M$ unit matrix $\mathbb{1}_{M}$ and the $2 \times 2$ matrix

$$
\mathscr{P}=\left(\begin{array}{cc}
\left\langle\operatorname{tr} \mathscr{G}^{e e}\right\rangle & -\left\langle\operatorname{tr} \mathscr{G}^{e h}\right\rangle \\
-\left\langle\operatorname{tr} \mathscr{G}^{h e}\right\rangle & \left\langle\operatorname{tr} \mathscr{G}^{h h}\right\rangle
\end{array}\right)
$$

We seek the solution of equations (17) and (18) which satisfies

$$
\varepsilon \mathscr{G}(\varepsilon) \rightarrow 1_{2 M} \text { if }|\varepsilon| \gg \lambda
$$

It is convenient to define a self energy

$$
\Sigma=\left(\begin{array}{ll}
\Sigma^{e e} & \Sigma^{e h} \\
\Sigma^{h e} & \Sigma^{h h}
\end{array}\right)=\frac{\lambda}{M}\left(\begin{array}{cc}
\operatorname{tr} \mathscr{G}^{e e} & \operatorname{tr} \mathscr{G}^{c h} \\
\operatorname{tr} \mathscr{G}^{h e} & \operatorname{tr} \mathscr{G}^{h h}
\end{array}\right)
$$

Equations (17)-(20) contain a closed set of equations from which $\langle\Sigma\rangle$ can be determined. 
We are interested in the limit $M \rightarrow \infty, \lambda \rightarrow \infty$, keeping $N$ and $\varepsilon / \delta=2 \varepsilon M / \lambda \pi$ fixed. In this limit, the equations for $\langle\Sigma\rangle$ become

$$
\begin{gathered}
\left\langle\Sigma^{e e}\right\rangle=\left\langle\Sigma^{h h}\right\rangle,\left\langle\Sigma^{e h}\right\rangle\left\langle\Sigma^{h e}\right\rangle-\left\langle\Sigma^{e e}\right\rangle^{2}=1, \\
\frac{\pi \varepsilon}{2 \delta}\left\langle\Sigma^{e h}\right\rangle+\sum_{j=1}^{N} K_{j}\left(\varepsilon\left\langle\Sigma^{e h}\right\rangle+\Delta \mathrm{e}^{\mathrm{d} \Phi},\left\langle\Sigma^{e e}\right\rangle\right)=0, \\
\frac{\pi \varepsilon}{2 \delta}\left\langle\Sigma^{h e}\right\rangle+\sum_{j=1}^{N} K_{j}\left(\varepsilon\left\langle\Sigma^{h e}\right\rangle+\Delta \mathrm{e}^{-1 \Phi^{\prime \prime}}\left\langle\Sigma^{e e}\right\rangle\right)=0 .
\end{gathered}
$$

The function $K_{f}(\varepsilon)$ is defined through

$$
\left.\Gamma_{J} / K_{J}=\left(4-2 \Gamma_{J}\right) \sqrt{\Delta^{2}-\varepsilon^{2}}+\Gamma_{J}\left(\Delta \mathrm{e}^{i \Phi_{J}}\left\langle\Sigma^{e h}\right\rangle+\Delta \mathrm{e}^{-1 \Phi_{J} / \Sigma^{h e}}\right\rangle+2 \varepsilon\left\langle\Sigma^{e e}\right\rangle\right) .
$$

(We have substituted from equation (11) for the matrix $W$.) The boundary condition in equation (19) becomes ineffective in the limit $\lambda \rightarrow \infty$. Instead, we seek the solution of equations (21) with $\left\langle\Sigma^{e e}\right\rangle=\left\langle\Sigma^{h h}\right\rangle \rightarrow-\mathrm{i}$ for $\varepsilon \rightarrow \mathrm{i} \infty$, corresponding to a constant density of states $\rho(\varepsilon)=1 / \delta$ for $|\varepsilon| \gg \Delta$. From $\langle\Sigma\rangle$, we find $\langle\mathscr{G}\rangle$ and hence the ensemble averaged supercurrent $\langle I\rangle$ is found to be

$$
\langle I\rangle=\frac{2 e}{\mathrm{i} \hbar} k T \Delta \sum_{n=0}^{\infty} \sum_{j=1}^{N} \operatorname{sign}\left(\Phi_{\prime \prime}\right) K_{J}\left[\mathrm{e}^{\mathrm{i} \Phi_{\mu}}\left\langle\Sigma^{h e}\left(\mathrm{i} \omega_{n}\right)\right\rangle-\mathrm{e}^{-I \Phi_{\prime \prime}}\left\langle\Sigma^{e h}\left(\mathrm{i} \omega_{n}\right)\right\rangle\right] .
$$

Equations (21) and (22) contain all the information needed to determine the average supercurrent through a chaotic Josephson junction.

An analytic solution of equations (21) is possible in certain limiting cases. Here we discuss the case of high tunnel barriers, $\Gamma_{J} \ll 1$, for all $j$. Then we may approximate $K_{l}=$ $(1 / 4) \Gamma,\left(\Delta^{2}-\varepsilon^{2}\right)^{-1 / 2}$ and find

$$
\begin{aligned}
\left\langle\Sigma^{e e}\right\rangle & =\left\langle\Sigma^{h h}\right\rangle=-\varepsilon\left(\sqrt{\Delta^{2}-\varepsilon^{2}}+E_{\mathrm{T}}\right)\left[|\Omega|^{2} \Delta^{2}-\varepsilon^{2}\left(\sqrt{\Delta^{2}-\varepsilon^{2}}+E_{\mathrm{T}}\right)^{2}\right]^{-1 / 2}, \\
\left\langle\Sigma^{e h}\right\rangle & =\Omega \Delta\left[|\Omega|^{2} \Delta^{2}-\varepsilon^{2}\left(\sqrt{\Delta^{2}-\varepsilon^{2}}+E_{\mathrm{T}}\right)^{2}\right]^{-1 / 2}, \\
\left\langle\Sigma^{h e}\right\rangle & =\Omega^{*} \Delta\left[|\Omega|^{2} \Delta^{2}-\varepsilon^{2}\left(\sqrt{\Delta^{2}-\varepsilon^{2}}+E_{\mathrm{T}}\right)^{2}\right]^{-1 / 2}, \\
\Omega(\phi) & =\frac{\delta}{2 \pi} \sum_{j=1}^{N} \Gamma_{j} e^{\mathrm{e}^{\Phi}{ }_{n},} E_{\mathrm{T}}=\frac{\delta}{2 \pi} \sum_{j=1}^{N} \Gamma_{j}=\Omega(0) .
\end{aligned}
$$

The energy $E_{\mathrm{T}}$ is related to the mean dwell-time through $E_{\mathrm{T}}=\hbar / 2 \tau_{\text {dwell }}$. (The dwell-time is defined as $\tau_{\text {dwell }}=(\hbar / \mathrm{i} N)(\partial / \partial \varepsilon)(\ln \operatorname{det} S(\varepsilon))$, see Ref. [33].) The excitation gap in the spectrum of the Josephson junction is of order $|\Omega(\phi)|$ when $\tau_{\text {dwell }} \gg \hbar / \Delta$ [18]. Substitution from the above equations into equation (22) yields the supercurrent

$$
\langle I\rangle=\frac{2 \pi}{e} k T G E_{\mathrm{T}} \sum_{n=0}^{\infty} \frac{\Delta^{2} \sin \phi}{\sqrt{\Delta^{2}+\omega_{n}^{2}}}\left[|\Omega|^{2} \Delta^{2}+\omega_{n}^{2}\left(\sqrt{\Delta^{2}+\omega_{n}^{2}}+E_{\mathrm{T}}\right)^{2}\right]^{-1 / 2},
$$

where

$$
G=\frac{2 e^{2}}{h} \frac{\sum_{t=1}^{N_{1}} \sum_{j=N_{1}+1}^{N} \Gamma_{t} \Gamma_{J}}{\sum_{k=1}^{N} \Gamma_{k}}
$$

is the conductance of the Josephson junction when the superconductors are in the normal state.

For arbitrary transmission probabilities $\Gamma_{\rho}$, it is necessary to solve equations (21) 


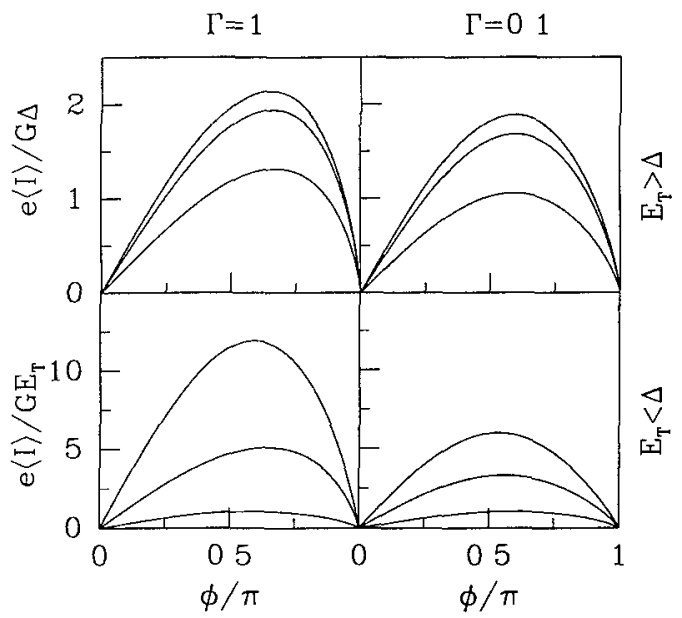

Fig. 2. Average supercurrent at zero temperature, computed from equations (21) and (22) for the case $N_{1}=N_{2}=N / 2, \Gamma=\Gamma$, for all $J$. Left panels $\Gamma=1$; right panels $\Gamma=0.1$. The upper panels show $\langle I\rangle$ in the short dwell-tıme regime for $E_{\Gamma} / \Delta=1$ (bottom curve), 10 and 100 (top curve). The bottom panels show $\langle I\rangle$ in the long dwell-tıme regime for $E_{\mathrm{T}} / \Delta=0.01$ (top curve), 0.1 and 1 (bottom curve). The conductance $G=\left(2 e^{2} / h\right) N \Gamma / 4$ and the Thouless energy $E_{\mathrm{T}}=N \Gamma \delta / 2 \pi$. Notice that $\langle I\rangle$ is in units of $G \Delta / e$ in the top panels and in units of $G E_{\mathrm{T}} / e$ in the bottom panels.

numerically. We have studied the case when both point contacts have an equal number of modes $\left(N_{1}=N_{2}=N / 2\right)$, and that all transmission probabilities are equal $\left(\Gamma_{J}=\Gamma\right.$, for all $\left.\jmath\right)$. The average supercurrent at zero temperature for $\Gamma=0.1$ and $\Gamma=1$ is shown in Fig. 2 .

\section{ERGODIC REGIME}

The general result in equation (22) describes the supercurrent in the ergodic regime $\tau_{\text {erg }} \ll \hbar / \Delta$. Within this regime, we can distinguish two further regimes, depending on whether the dwell time $\tau_{\text {dwell }}=\hbar / 2 E_{\mathrm{T}}$ is short or long compared with $\hbar / \Delta$. We discuss these two regimes in two separate subsections.

\subsection{Short dwell-time regime}

In the short dwell-time regime (when $\tau_{\mathrm{dwell}} \ll \hbar / \Delta$ or, equivalently, $E_{\mathrm{T}} \gg \Delta$ ), the magnitude of the critical current $I_{\mathrm{c}}=\max _{\phi} I(\phi)$ is set by the energy gap $\Delta$ in the bulk superconductor: $I_{\mathrm{c}} \approx G \Delta / e$ at zero temperature. The temperature dependence of $I_{\mathrm{c}}$ can be neglected as long as $k T \ll \Delta$, i.e. for temperatures $T$ much less than the critical temperature $T_{\mathrm{c}}$ of the bulk superconductor. In the case of tunneling contacts, evaluation of equation (24) with $E_{\mathrm{T}} \gg \Delta \gg k T$ yields

$$
\langle I\rangle=\frac{G \Delta}{e} \frac{K(\gamma \sin (\phi / 2)) \sin \phi}{\sqrt{1-\gamma^{2} \sin ^{2}(\phi / 2)}} .
$$

The conductance $G$ was defined in equation (25), the function $K$ is the complete elliptic integral of the first kind, and we abbreviated

$$
\gamma=2\left(\sum_{\imath=1}^{N_{1}} \sum_{J=N_{1}+1}^{N} \Gamma_{\imath} \Gamma_{J}\right)^{1 / 2}\left(\sum_{k=1}^{N} \Gamma_{k}\right)^{-1} .
$$

The parameter $\gamma$ equals 1 for two identical point contacts with mode-independent tunnel probabilities. 
The result in equation (26) could also have been obtained directly from the general formula for the zero-temperature supercurrent in the short dwell-time regime [19]

$$
\langle I\rangle=\frac{e \Delta}{2 \hbar} \int_{0}^{1} \mathrm{~d} t \rho(t) \frac{t \sin \phi}{\sqrt{1-t \sin ^{2}(\phi / 2)}},
$$

which relates $\langle I\rangle$ to an integral over the transmission eigenvalues $t$ of the junction in the normal state, with density $\rho(t)$. The transmission eigenvalues density for a chaotic cavity with two identical tunneling contacts $\left(N_{1}=N_{2}=N / 2, \Gamma_{j}=\Gamma_{j+N / 2}\right.$, for $\left.j=1,2, \ldots, N / 2\right)$ is given [34] by

$$
\rho(t)=\sum_{j=1}^{N / 2} \frac{\Gamma_{j}\left(2-\Gamma_{j}\right)}{\pi\left(\Gamma_{j}^{2}-4 \Gamma_{j} t+4 t\right) \sqrt{t(1-t)}} .
$$

One can check that equation (28) agrees with equation (26) with $\gamma=1$ if $\Gamma_{j} \ll 1$, for all $j$. For two identical ballistic point contacts $\left(N_{1}=N_{2}=N / 2, \Gamma_{,}=1\right.$, for all $\left.j\right)$, the density is $\rho(t)=N(2 \pi)^{-1}[t(1-t)]^{-1 / 2}[35,36]$, which yields

$$
\langle I\rangle=\frac{2 e \Delta}{\mathrm{i} \pi \hbar \sin (\phi / 2)} G E(\mathrm{i} \operatorname{arsinh}[\tan (\phi / 2)], \mathrm{i} \operatorname{cotan}(\phi / 2)) .
$$

Here $G=N / 4$ and $E$ is the elliptic integral of the second kind.

\subsection{Long dwell-time regime}

In the long dwell-time regime (when $\tau_{\text {dwcll }} \gg \hbar / \Delta$ or, equivalently, $E_{\mathrm{T}} \ll \Delta$ ), the magnitude of the critical current is set by the Thouless energy, but retains a logarithmic dependence on $\Delta$, so that $I_{\mathrm{c}} \simeq\left(G E_{\mathrm{T}} / e\right) \ln \left(\Delta / E_{\mathrm{T}}\right)$. The temperature dependence of $I_{\mathrm{c}}$ can be neglected so long as $k T \ll E_{\mathrm{T}}$. If $k T \gg E_{\mathrm{T}}$ (but still $T \ll T_{\mathrm{c}}$ ) the critical current decreases, though only logarithmically, so that $I_{\mathrm{c}} \simeq\left(G E_{\mathrm{T}} / e\right) \ln (\Delta / k T)$. For the case of tunneling contacts, we find from equation (24) the expressions

$$
\begin{aligned}
& \langle I\rangle=\frac{G E_{\mathrm{T}}}{e} \sin \phi \ln \left(\frac{2 \Delta / E_{\mathrm{T}}}{\sqrt{1-\gamma^{2} \sin ^{2}(\phi / 2)}}\right), k T \ll E_{\mathrm{T}}, \\
& \langle I\rangle=\frac{G E_{\mathrm{T}}}{e} \sin \phi\left[\ln \left(\frac{2 \Delta}{\pi k T}\right)+c_{\text {Euler }}\right], k T \gg E_{\mathrm{T}}, T \ll T_{\mathrm{c}},
\end{aligned}
$$

where $c_{\text {Euler }} \approx 0.58$ is Euler's constant. For ballistic contacts, we do not have such simple expressions as these, but the parametric dependence of $I$ on $\Delta, E_{\mathrm{T}}$ and $k T$ is the same as for tunneling contacts (see Fig. 2).

The logarithmic dependence on $\Delta$ of the supercurrent when $E_{\mathrm{T}} \ll \Delta$ arises because the Thouless energy $E_{\mathrm{T}}$ is not an effective cutoff for the Matsubara sum in equation (8) or, equivalently, for the energy integration of equation (7). Spectral correlations exist up to energies of order $\hbar / \tau_{\text {erg }} \gg E_{\mathrm{T}}$. These long-range spectral correlations are responsible for the weak decay $\Sigma^{e h} \propto 1 / \omega$ of the self-energy and $\rho-\delta^{-1} \propto 1 / \varepsilon^{2}$ of the density of states. The superconducting energy gap $\Delta$ has to serve as a cutoff energy for the otherwise logarithmically divergent equations (7) and (8), which explains the logarithm $\ln \Delta$ in equations (31a) and (31b).

\section{NON-ERGODIC REGIME}

When $\tau_{\mathrm{dwell}} \geq \hbar / \Delta$, a random-matrix theory of the Josephson effect is no longer possible, because the non-ergodic dynamics on time scales shorter than $\tau_{\text {erg }}$ start to play a role. To 
study the average supercurrent in this non-ergodic regime, we return to equation (8). On substitution of equations (1) and (2), we obtain an expression for $I$ in terms of the scattering matrix $S$ of the normal region

$$
\begin{aligned}
I & =\frac{2 \pi}{e} k T \sum_{n=0}^{\infty} F\left(\omega_{n}\right), \\
F(\omega) & =-\frac{4 e^{2}}{h} \frac{\mathrm{d}}{\mathrm{d} \phi} \operatorname{tr} \ln \left[1-\alpha(\mathrm{i} \omega)^{2} S(\mathrm{i} \omega) \mathrm{e}^{\mathrm{I} \Phi} S^{*}(-\mathrm{i} \omega) \mathrm{e}^{-\mathrm{I}^{\top}}\right] .
\end{aligned}
$$

The evaluation of the scattering matrix at the imaginary energy $i \omega_{n}$ is equivalent to the evaluation of the scattering matrix at the Fermi level in the presence of absorption, with rate $1 / \tau_{\mathrm{abs}}=2 \omega_{n} / \hbar=(2 n+1) 2 \pi k T / \hbar$. We first consider temperatures $k T \gg E_{\mathrm{T}}$. Since $\omega_{n} \gg$ $E_{\mathrm{T}}=\hbar / 2 \tau_{\mathrm{dwell}}$ for all $n$ in this high temperature regime, absorption is strong, $\tau_{\mathrm{abs}} \ll \tau_{\mathrm{dwell}}$. The formal correspondence between Matsubara frequency and absorption rate helps to understand that, to lowest order in $\tau_{\mathrm{abs}} / \tau_{\mathrm{dwell}}=E_{\mathrm{T}} / \omega$, the diagonal elements of $S(\mathrm{i} \omega)$ are given by the reflection amplitudes of the tunnel barriers in the contacts, $S_{J J}=\left(1-\Gamma_{\jmath}\right)^{1 / 2}$, while the off-diagonal elements satisfy

$$
\left\langle\left|S_{\imath}(i \omega)\right|^{2}\right\rangle=\frac{\Gamma_{t} \Gamma_{,}}{\sum_{k=1}^{N} \Gamma_{k}} \int_{0}^{\infty} \mathrm{d} \tau P_{\imath \jmath}(\tau) \exp (-2 \omega \tau / \hbar), \quad i \neq j .
$$

The function $P_{l j}$ is the classical distribution of dwell times for particles that enter the quantum dot through mode $j$ and exit through mode $i$. Because of the smallness of $\left\langle\left|S_{l j}(i \omega)\right|^{2}\right\rangle=\mathrm{O}\left(E_{\mathrm{T}} / \omega\right)$, it is sufficient to keep only the lowest order term in an expansion of $\langle F(\omega)\rangle$ in the off-diagonal scattering matrix elements,

$$
\langle F(\omega)\rangle=-\frac{4 e^{2}}{h} \sum_{t=1}^{N_{1}} \sum_{J=N_{i}+1}^{N} \frac{2 \alpha(\mathrm{i} \omega)^{2}\left\langle\left|S(\mathrm{i} \omega)_{l}\right|^{2}\right\rangle \sin \phi}{\left[1-\alpha(\mathrm{i} \omega)^{2}\left(1-\Gamma_{l}\right)\right]\left[1-\alpha(\mathrm{i} \omega)^{2}\left(1-\Gamma_{\jmath}\right)\right]} .
$$

Equations (32a), (32b), (33) and (34) permit a semiclassical calculation of the average supercurrent in the non-ergodic regime for temperatures $k T \gg E_{\mathrm{T}}$, where random-matrix theory fails. The only input required is the classical distribution of dwell times.

On time scales greater than $\tau_{\text {erg }}$, the distribution $P_{l j}$ is exponential with the same mean dwell time $\tau_{\mathrm{dwcll}}=\hbar / 2 E_{\mathrm{T}}$, for all $i, j$ :

$$
P_{l J}(\tau)=\frac{2 E_{\mathrm{T}}}{\hbar} \exp \left(-2 E_{\mathrm{T}} \tau / \hbar\right)
$$

The non-chaotic dynamics on time scales shorter than $\tau_{\mathrm{erg}}$ enter through a non-universal form of $P_{l}$ for $\tau \leqslant \tau_{\mathrm{erg}}$. We consider the case of a ballistic dynamics (size $L$ of the normal region much less than the mean free path $\ell$ ). The ergodic time $\tau_{\text {crg }} \simeq L / v_{\mathrm{F}}$ is then a lower cutoff on $P_{y j}$, since the minimum dwell time $L / v_{\mathrm{F}}$ is the time needed to cross the system ballistically. A qualitative estimate of $\langle I\rangle$ is obtained if we set $P_{l y}(\tau)=0$ for $\tau<L / v_{\mathrm{F}}$ and approximate it by equation (35) for larger times. Substitution of this dwell-time distribution into equation (33) gives

$$
\left\langle\left|S_{i j}(i \omega)\right|^{2}\right\rangle=\frac{\Gamma_{t} \Gamma_{j}}{\sum_{k=1}^{N} \Gamma_{h}} \frac{E_{\mathrm{T}}}{\omega} \exp \left(-2 \omega L / \hbar v_{\mathrm{F}}\right), \omega \gg E_{\mathrm{T}}, \quad i \neq j .
$$


We next compute $\langle F(\omega)\rangle$ from equation (34), replacing $\alpha(\mathrm{i} \omega)$ by its value $-\mathrm{i}$ for $\omega \ll \Delta$. The result is

$$
\begin{aligned}
\langle F(\omega)\rangle & =\frac{\tilde{G} E_{\mathrm{T}}}{\omega} \exp \left(-2 \omega L / \hbar v_{\mathrm{F}}\right) \sin \phi, \\
\tilde{G} & =\frac{2 e^{2}}{h} \frac{\sum_{t=1}^{N_{1}} \sum_{j=N_{1}+1}^{N} \Gamma_{t} \Gamma_{l}\left(2-\Gamma_{t}\right)^{-1}\left(2-\Gamma_{t}\right)^{-1}}{\sum_{k=1}^{N} \Gamma_{k}} .
\end{aligned}
$$

Notice that $\tilde{G}=G$ for the case of high tunnel barriers $\left(\Gamma_{J} \ll 1\right.$, for all $\left.j\right)$. We can now calculate the average supercurrent from equations (32a) and (32b). Equation (37a) is valid for $E_{\mathrm{T}} \ll \omega \ll \Delta, E_{\mathrm{T}} \ll \hbar v_{\Gamma} / L \ll \Delta$, and is sufficient to determine the supercurrent in the temperature range $E_{\mathrm{T}} \ll k T \ll \Delta$. Substitution from equation (37) into equation (32) gives

$$
\begin{aligned}
& \langle I\rangle=\frac{\tilde{G} E_{\mathrm{T}}}{e} \sin \phi \ln \left(\hbar v_{\mathrm{F}} / \pi k T L\right), E_{\mathrm{T}} \ll k T \ll \hbar v_{\mathrm{F}} / L \ll \Delta, \\
& \langle I\rangle=\frac{\tilde{G} E_{\mathrm{T}}}{e} 2 \sin \phi \exp \left(-2 \pi k T L / \hbar v_{\mathrm{F}}\right), E_{\mathrm{T}} \ll \hbar v_{\mathrm{F}} / L \ll k T \ll \Delta .
\end{aligned}
$$

Equations (38a) and (38b) have the same temperature dependence as the result of Ref. [11] for the double-barrier SNS junction.

We now turn to low temperatures $k T \leqslant E_{\mathrm{T}}$. In this temperature regime, the Matsubara sum in equation (32) contains terms with $\omega_{n} \lesssim E_{\mathrm{T}}$, for which the off-diagonal scattering matrix elements $S_{l j}\left(\mathrm{i} \omega_{n}\right)$ are not small and the approximation of equation (34) is no longer valid. However, since $E_{\mathrm{T}} \ll \hbar / \tau_{\text {crg }}$, these Matsubara frequencies are well within the validity range of random-matrix theory. Therefore, we can use the results of Section 3 to compute $\langle F(\omega)\rangle$ for $\omega \lesssim E_{\mathrm{T}}$ and the semiclassical formula in equation (34) for $\omega \gtrsim E_{\mathrm{T}}$. These two results match at $\omega \simeq E_{\mathrm{T}}$, because the validity range $\omega \ll \hbar / \tau_{\mathrm{erg}}$ of random-matrix theory and the validity range $\omega \gg E_{\mathrm{T}}$ of the semiclassical theory overlap (assuming $\tau_{\mathrm{erg}} \ll \tau_{\mathrm{dwcll}}=$ $\left.\hbar / 2 E_{\mathrm{T}}\right)$.

For the case of high tunnel barriers, random-matrix theory gives [cf. equation (24)]

$$
\langle F(\omega)\rangle=\frac{G E_{\mathrm{T}} \sin \phi}{\sqrt{|\Omega(\phi)|^{2}+\omega^{2}}}, \omega \ll \hbar v_{\mathrm{F}} / L \ll \Delta,
$$

while the semiclassical formula of equation (34) gives

$$
\langle F(\omega)\rangle=\frac{G E_{\mathrm{T}} \sin \phi}{\omega} \exp \left(-2 \omega L / \hbar v_{\mathrm{F}}\right), E_{\mathrm{T}} \ll \omega \ll \Delta .
$$

(The function $\Omega(\phi)$ was defined in equation (23d).) The two results in equations (39) and (40) have a common range of validity $E_{\mathrm{T}} \ll \omega \ll \hbar v_{\mathrm{F}} / L$ within which they can be matched. The result is a formula valid for all $\omega \ll \Delta$, for a ballistic quantum dot with high tunnel barriers:

$$
\langle F(\omega)\rangle=\frac{G E_{\mathrm{T}} \sin \phi}{\sqrt{|\Omega(\phi)|^{2}+\omega^{2}}} \exp \left(-2 \omega L / \hbar v_{\mathrm{F}}\right), \omega \ll \Delta .
$$

After substitution from equation (41) into equation (32) we obtain the average supercurrent in the low-temperature regime

$$
\langle I\rangle=\frac{G E_{\mathrm{T}}}{e} \sin \phi\left[\ln \left(\frac{\hbar v_{\mathrm{F}}}{L E_{\mathrm{T}} \sqrt{1-\gamma^{2} \sin ^{2}(\phi / 2)}}\right)-c_{\text {Euler }}\right], k T \ll E_{\mathrm{T}} \ll \hbar v_{\mathrm{F}} / L \ll \Delta .
$$


Table 1 Parametric dependence of the zero-temperature critical current $I_{c}$ on the three time scales $\tau_{\mathrm{cdw} \text { ill }}, \tau_{\mathrm{crg}}$ and $\hbar / \Delta$ The Thouless energy $E_{\mathrm{T}}=\hbar / 2 \tau_{\text {dwell }}$

\begin{tabular}{|c|c|c|c|}
\hline \multirow[t]{2}{*}{$I_{c} \times e / G$} & \multicolumn{2}{|c|}{ Weak couplıng $\left(\tau_{\mathrm{dwell}} \gg \tau_{\text {crg }}\right)$} & \multirow{2}{*}{$\begin{array}{l}\text { Strong coupling } \\
\left(\tau_{\text {dwell }} \simeq \tau_{\mathrm{tr}}\right)\end{array}$} \\
\hline & Ergodic $\left(\tau_{\mathrm{elg}} \ll \hbar / \Delta\right)$ & Non-ergodic $\left(\tau_{\mathrm{cI}} \gg \hbar / \Delta\right)$ & \\
\hline $\begin{array}{l}\text { Short dwell time }\left(\tau_{\text {dwell }} \ll \hbar / \Delta\right) \\
\text { Long dwell time }\left(\tau_{\text {dwell }} \gg \hbar / \Delta\right)\end{array}$ & $\stackrel{\Delta}{E_{\mathrm{T}} \ln \left(\Delta / E_{\mathrm{T}}\right)}$ & $E_{\mathrm{I}} \ln \left(\hbar / E_{\mathrm{T}} \tau_{\mathrm{clg}}\right)$ & $\stackrel{\Delta}{E_{\mathrm{T}}}$ \\
\hline
\end{tabular}

(The parameter $\gamma$ was defined in equation (27).) The results in equations (38) and (42) cover the entire temperature range below $T_{\mathrm{c}}$.

\section{CONCLUSIONS}

In Table 1, we summarize the parametric dependence of the critical current at zero temperature on the three time scales $\tau_{\text {dwell }}, \tau_{\text {erg }}$ and $\hbar / \Delta$. We show the three new regimes for a weakly coupled normal region $\left(\tau_{\text {dwell }} \gg \tau_{\text {erg }}\right)$, and have included for comparison also the two old regimes for a strongly coupled normal region $\left(\tau_{\mathrm{dwell}} \simeq \tau_{\mathrm{crg}}\right)$. Apart from a logarithmıc factor, the critical current is given by $I_{\mathrm{c}} \simeq(G / e) \min \left(\hbar / \tau_{\mathrm{dwcll}}, \Delta\right)$ in each of the five regimes. There is an additional logarithmic dependence on $\min \left(\tau_{\mathrm{dwell}} / \tau_{\mathrm{erg}}, \tau_{\mathrm{dwcl1}} \Delta / \hbar\right)$ in two of the three new regimes. Upon raising the temperature, the critical current is suppressed at a characteristic temperature given by $\min \left(\hbar / \tau_{\text {crg }}, \Delta\right)$. At lower temperatures, $I_{c}$ has a logarithmic $T$-dependence so long as $T \geq \hbar / \tau_{\mathrm{dwell}}$ and becomes $T$-independent at still lower $T$.

In this work, we did not address the sample-to-sample fluctuations of the supercurrent, but calculated only the ensemble average. For strongly coupled diffusive Josephson junctions ( $\tau_{\text {dwell }} \simeq \tau_{\text {erg }}, L \gg \ell$ ), the root-mean-squared of the fluctuations is a factor $e^{2} / h G$ smaller than the average critical current $[19,37]$. Prelıminary calculations in the ergodic regime indicate that the same is true for weakly coupled Josephson junctions $\left(\tau_{\mathrm{dwcll}} \gg \tau_{\mathrm{crg}}\right)$, 1.e. the r.m.s. fluctuations of $I_{c}$ are given by the entries in Table 1 multiplied by $e / h$.

We close with a remark on quantum dots with an integrable classical dynamics, such as rectangular or circular ballistic cavities. For energies $\varepsilon \lesssim E_{\mathrm{T}}$, the excitation spectrum of an integrable Josephson junction is quite different from its chaotic counterpart [18]. The density of states $\rho(\varepsilon)$ of a chaotic cavity in contact with a superconductor shows a gap of size $E_{\mathrm{T}}$ around the Fermı level $\varepsilon=0$, while $\rho(\varepsilon)$ vanishes linearly when $\varepsilon \rightarrow 0$ for a rectangular or circular cavity It is an interesting open problem to compute the supercurrent through an integrable cavity and compare with the results for the chaotic case obtained in this paper.

Acknowledgements-We thank A I Larkın and Yu N Ovchınnıkov for alertıng us to the logarithmıc temperature dependence of the supercurrent found in Ref [11] This work was supported by the 'Stıchtıng voor Fundamenteel Onderzoek der Materie' (FOM) and by the Nederlandse organısatie voor Wetenschappelıjk Onderzoek (NWO)

\section{REFERENCES}

1 Marsh, A M, Williams, D A and Ahmed, H, Phys Rev B, 1994, 50, 8118

2 Takayanagı, H, Akazakı, T and Nitta, J, Phys Rev B, 1995, 51, 1374

3 Courtols, H, Gandit, Ph and Pannetier, B, Phys Rev B, 1995, 51, 9360 1995, 52, 1162

4 Courtoıs, H, Gandit, Ph, Mailly, D and Pannetıer, B, Phys Rev Lett, 1996, 76, 130

5 Charlat, P, Courtois, H, Gandit, Ph, Mailly, D, Volkov, A F and Pannetier, B, preprint (cond mat/9609182) 
6 Mur, L C, Harmans, C J P M, Mooı, J E, Carlın, J F, Rudra, A and Ilegems, M, Phys Rev B, 1996, 54, 2327

7 Volkov, A F, Magnee, P H C, van Wees, B J and Klapwijk, T M, Phys C, 1995, 242, 261

8 Volkov, A F and Takayanag1, H, Phys Rev Lett, 1996, 76, 4026, Phys Rev B, to be published

9 Wilhelm, F K, Zaikın, A D and Schon, G, J Low Temp Phys 1997 106, 305

10 Kulık, I O, Zh Eksp Teor Flz, 1969, 57, 1745 (Sov Phys JETP, 1970, 30, 944)

11 Aslamasov, L G, Larkın, A I and Ovchınnikov, Yu N, Zh Eksp Teor FIz, 1968, 55, 323 (Sov Phys JETP, 1969, 28, 171)

12 van Wees, B J and Takayanag1, H, In Mesoscopic Electron Transport, ed L P Kouwenhoven, G Schon and L L Sohn NATO ASI Series E, Kluwer, Dordrecht, to be published

13 Efetov, K B , Adv Phys, 1983, 32, 53

14 Andreev, A V, Agam, O, Simons, B D and Altshuler, B L, Phys Rev Lett, 1996, 76, 3967

15 Altshuler, B L and Shklovskit, B I, Zh Eksp Teor Flz, 1986, 91, 220 (Sov Phys JETP, 1986, 64, 127)

16 Likharev, K K, Rev Mod Phys, 1979, 51, 101

17 Svidzınskı, A B, Antsygina, T N and Bratus', E N, Zh Eksp Teor Fiz, 1971, 61, 1612 (Sov Phys JETP, 1972, 34, 860)

18 Melsen, J A, Brouwer, P W, Frahm, K M and Beenakker, C W J, Europhys Lett, 1996, 35, 7, Phys Scripta, to be published (cond-mat/9607036), Phys Rev B, 1996, 54, 9443

19 Beenakker, C W J , Phys Rev Lett, 1991, 67, 3836, 1992, 68, 1442 (E)

20 Beenakker, C W J, In Transport Phenomena in Mesoscopıc Systems, ed H Fukuyama and T Ando Springer, Berlin, 1992

21 Andreev A F, Zh Eksp Teor $F_{l z}, 1964,46,1823$ (Sov Phys JETP, 1964, 19, 1228)

22 Doron, E and Smilansky, U, Phys Rev Lett, 1992, 68, 1255

23 Beenakker, C W J and van Houten, H In Nanostructures and Mesoscopic Systems, ed W P Kırk Academıc, New York, 1992

24 Mahaux, C and Weidenmuller, H A, Shell-Model Approach to Nuclear Reactons North-Holland, Amsterdam, 1969

25 Verbaarschot, J J M, Weidenmuller, H A and Zirnbauer, M R, Phys Rep, 1985, 129, 367

26 Mehta, M L, Random Matrices Academıc, New York, 1991

27 Iida, S, Weidenmuller, H A and Zuk, J A, Phys Rev Lett, 1990, 64, 583, Ann Phys (N Y), 200, 1990,219

28 Brouwer, P W, Phys Rev B, 1995, 51, 16878

29 Frahm, K M, Brouwer, P W, Melsen, J A and Beenakker, C W J, Phys Rev Lett, 1996, 76, 2981

30 Pandey, A, Ann Phys (N Y), 1981, 134, 110

31 Brézın, E and Zee, A, Phys Rev E, 1994, 49, 2588

32 Pastur, L A, Teor Mat Flz, 1972, 10, 102, Theor Math Phys, 1972, 10, 67

33 Wigner, E P, Phys Rev, 1955, 98, 145, Smith, F T, Phys Rev, 1960, 118, 349

34 Brouwer, P W and Beenakker, C W J , J Math Phys, 1996, 37, 4904

35 Baranger, H U and Mello, P A, Phys Rev Lett, 1994, 73, 142

36 Jalabert, R A, Pichard, J L and Beenakker, C W J, Europhys Lett, 1994, 27, 255

37 Altshuler, B L and Spivak, B Z, Zh Eksp Teor Flz, 1987, 92, 609 (Sov Phys JETP, 1987, 65, 343) 